\title{
Golden ratio and the proportionality between pulmonary pressure components
}

\section{in pulmonary arterial hypertension}

First author's surname: Chemla. Running head: Pulmonary pressure and the Golden Ratio

Denis Chemla, MD, David Boulate*, MD, Jason Weatherald, MD, Edmund M.T. Lau, MD, Pierre Attal, MD, Laurent Savale, MD, David Montani, MD, Elie Fadel, MD, MD, Olaf Mercier, MD, Olivier Sitbon, MD, Marc Humbert, MD, and Philippe Hervé, MD.

*Denis Chemla and David Boulate contributed equally to the study.

Word count Body of the text: 2,499 Abstract: 249

37 references / 6 Tables / 3 Figures / Data Supplements ESM

\section{Affilitations}

Service d'explorations fonctionnelles multidisciplinaires bi-site Antoine Béclère - Kremlin Bicêtre, APHP, Le Kremlin-Bicêtre, France (DC); Université Paris-Sud, Faculté de Médecine, Université ParisSaclay, Le Kremlin-Bicêtre, France (DC, LS, DM, OS, MH); INSERM UMR_S 999, Hôpital Marie Lannelongue, Le Plessis Robinson, France (DC, LS, DM, OS, MH, PH); Department of Thoracic and Vascular Surgery, Marie Lannelongue Hospital, Le Plessis-Robinson, France (DB, EF, OM).University of Calgary, Department of Medicine, Division of Respirology, and Libin Cardiovascular Institue of Alberta, Calgary, Alberta, Canada (JW); Department of Respiratory Medicine, Royal Prince Alfred Hospital, University of Sydney, Missenden Road, Camperdown, NSW 2050, Australia (EMTL); Department of Otolaryngology-Head and Neck Surgery, Shaare-Zedek Medical Center and Hebrew University Medical School, Jerusalem, Israel (PA); Service de Pneumologie, Hôpital Bicêtre, AP-HP, Le Kremlin-Bicêtre, France (LS, DM, OS, MH); Departement de Chirurgie Thoracique, Vasculaire et de Transplantation Pulmonaire, Hopital Marie Lannelongue, Le Plessis Robinson, France (PH).

Address correspondence to: Pr Denis CHEMLA, MD

Service des Explorations Fonctionnelles - Broca 4 Hôpital de Bicêtre, 78 rue du Général Leclerc, 94275 Le Kremlin Bicêtre, France

Email: denis.chemla@aphp.fr

Financial/non financial disclosures: None declared for all authors in the subject matter (pathophysiology)

Role of sponsors: None 


\section{ABBREVIATIONS LIST}

CTEPH: chronic thromboembolic pulmonary hypertension

dPAP: diastolic pulmonary artery pressure

mPAP: mean pulmonary artery pressure

PA: pulmonary artery

PAH: pulmonary arterial hypertension

PApp: pulmonary artery pulse pressure

PVR: pulmonary vascular resistance

RC-time: time constant of the PAP decay in diastole

sPAP: systolic pulmonary artery pressure

SV: stroke volume

$\Phi:$ Phi, the golden ratio 


\section{ABSTRACT (249 words)}

BACKGROUND: The golden ratio ( $\mathrm{Phi}, \Phi=1.618$ ) is a proportion that has been found in many phenomena in nature including the cardiovascular field. We tested the hypothesis that the systolic over mean pulmonary artery pressure ratio (sPAP/mPAP) and the mean over diastolic pressure ratio (mPAP/dPAP) may match $\Phi$ in patients with pulmonary arterial hypertension (PAH) and in controls.

METHODS: In the first, theoretical part of the study, we discussed why our hypothesis is consistent with three known hemodynamic features of the pulmonary circulation: (1) the 0.61 slope of the mPAP vs sPAP relationship, (2) pulmonary artery pulse pressure and mPAP have an almost 1:1 ratio, and (3) the proportional relationship between SPAP, MPAP and dPAP. In the second part, fluid-filled pressures were analyzed in 981 incident, untreated PAH and high-fidelity pressures were also analysed in 44 historical controls (mPAP range 9-113 $\mathrm{mmHg}$ ).

RESULTS: In PAH (non-normal distribution), median values of SPAP/mPAP and mPAP/dPAP were $1.591(98 \% \Phi)$ and $1.559(96 \% \Phi)$, respectively. In controls-(normal distribution), mean sPAP/dPAP and mPAP/dPAP were $1.572(97 \% \Phi)$ and $1.470(91 \% \Phi)$. In both PAH and controls, this was consistent with the $\Phi$ hypothesis assuming $<1 \mathrm{mmHg}$ error in estimation of sPAP, mPAP and dPAP on average.

CONCLUSIONS: In PAH and in controls, the fluctuations in SPAP and APAP around mPAP exhibited a constant scaling factor matched to $\Phi$. This remarkable property allows linkage of various empirical observations on pulmonary hemodynamics that were hitherto apparently unrelated. These findings warrant further confirmation in other types of $\mathrm{PH}$ and warrant explanation.

Key Words: pulmonary hypertension, hemodynamics 
The pulmonary circulation is a low pressure, low resistance and high capacitance system as compared to the systemic circulation. Pulmonary hypertension $(\mathrm{PH})$ is a pathophysiologic disorder defined by a mean pulmonary artery pressure (mPAP) $\geq 25 \mathrm{mmHg}$. PH complicates a number of heart and respiratory diseases, but other forms may be observed including idiopathic pulmonary arterial hypertension (1). PH is associated with worse prognosis regardless of etiology and there is thus a need to improve our understanding of pathophysiology of the pulmonary circulation.

Some striking hemodynamic features of the pulmonary circulation have been observed. The mPAP and systolic pulmonary artery pressure (sPAP) are strongly related through a linear relationship ( $\mathrm{mPAP}=0.61 \mathrm{sPAP}+2 \mathrm{mmHg}$ ) documented over a wide range of clinical and hemodynamic conditions $(2,3)$. This yet unexplained empirical relationhsip helps improve the noninvasive estimation of mPAP from the echocardiography-derived SPAP $(4,5)$. Another feature of the pulmonary circulation relates to PA pulse pressure (PApp), i.e., SPAP minus diastolic PA pressure (dPAP). Indeed, PApp and mPAP have an almost 1:1 ratio, while aortic pulse pressure is only half the mean aortic pressure (6). This is attested to the high pulsatility of the pulmonary circulation as compared to the systemic circulation.

A number of studies have reported that the golden ratio $(\mathrm{Phi}, \Phi)(7,8)$ may play a role in the cardiovascular field (9-15). Following the geometrical description made by the Greek Euclides more than two thousand years ago, $\Phi$ is the ratio of two quantities that fulfill strict criteria $(7,8) . \Phi$ is an irrational number approximately equal to 1.618 that allows replicating the same structure at different scales. The potential implication of $\Phi$ has been discussed in art, psychology and biology (717). It has also been suggested that the ratio between brachial systolic and diastolic blood pressure may be close to $\Phi$, at least in some patient subgroups $(10,14,15)$. However, the potential implication of $\Phi$ has not yet been studied at the pulmonary circulation level to our knowledge. In the present article, we tested the hypothesis that both the SPAP/mPAP ratio and the mPAP/dPAP ratio match $\Phi$ 
in a large population of patients with pulmonary arterial hypertension (PAH) of varying severity and in normotensive controls.

\section{METHODS}

\section{Theoretical background}

The golden ratio. Pythagoreans have described the propotional relationship between three quantities $a, b$ and $c$ as follows: $\mathrm{a} / \mathrm{b}=\mathrm{b} / \mathrm{c}$. Accordingly, $b^{2}=a \times c$, and $b$ is the geometric mean of $a$ and $c$ $(b=\sqrt{a \times c})$. Such proportional relationship may involve only two quantities in the special case where the term " $a$ " is in fact the sum of the two others $(a=b+c)(7)$. This was first geometrically described by Euclides who stated that "a straight line is said to have been cut in extreme and mean ratio when, as a whole line is to the greater segment, so is is the greater to the lesser" (The Elements, Chapt XIII). This property has been subsequently described algebraically as follows: $(b+c) / b=b / c=$ $\Phi=1.618034 \ldots .$. Among the numerous remarkable properties of the golden ratio, it must be recalled that $\Phi^{2}=\Phi+1=2.618$ and that $1 / \Phi=\Phi-1=0.618$. These properties explain why the golden ratio allows replicating the same structure at different scales (self-similarity) $(7,8)$.

Rationale for testing the golden ratio in the pulmonary circulation. Here we propose that there may be three reasons for testing the $\Phi$ hypothesis in the pulmonary circulation (Figure 1). The first one is that the slope of the mPAP vs SPAP linear relationship has been consistently reported to be $\sim 0.61$ over a wide range of MPAP, heart rate, cardiac output and PA wedge pressure, and irrespective of patient's age, sex and body mass index and of the cause of $\mathrm{PH}(2,3)$. Because the pressure intercept of this linear relationship is small, the mPAP/sPAP may be also close to $0.61(\sim 1 / \Phi)$, supporting the hypothesis that sPAP/mPAP matches $\Phi$. The second reason is that a previous systematic review of pulmonary hemodynamics obtained with high-fidelity pressure catheters has documented that there is a proportional relationship between SPAP, mPAP and dPAP $(\mathrm{mPAP}=\sqrt{\mathrm{SPAP} \times \mathrm{dPAP}})(18)$. The 
components of aortic pressure also follow this geometric rule (19). The third reason is that mPAP and PA pulse pressure have an almost 1:1 ratio (6). Thus, if $\mathrm{mPAP}=\mathrm{sPAP}-\mathrm{dPAP}$, this may be rewritten as follows: $\mathrm{SPAP}=\mathrm{mPAP}+\mathrm{dPAP}$. This equation is consistent with the condition governing the $\Phi$ hypothesis (see above, $a=b+c$ ) in cases where a propotional relationship applies (18).

\section{Patient Population and calculations}

This was an analysis of our fluid-filled pressure data $(n=981)$ contained in the retrospective study of incident, untreated patients from the French Pulmonary Arterial Hypertension Network registry who were enrolled between January 2006 and May 2016, were $\geq 18$ years of age and were diagnosed with idiopathic, heritable or drug-induced PAH on the basis of right heart catheterization results (20) (CNIL approval number 842063, May 24, 2003) (Tables 1 and 2). Because a small measurement error may have a tremendous impact on the two pressure ratios in subjects with low PA pressures, the hypothesis was tested on 44 controls (mPAP $\leq 20 \mathrm{mmHg}$ ) studied with high-fidelity pressure catheters (Table 3) extracted from our systematic review (18) involving five studies $(2,21$ 24). The unavoidable error in the estimation of the numerator and denominator of the ratios (i.e., PA pressures) was accounted for (17) (Table 4). Diagnosis of PAH, the catheterization technique, data analysis and statistics are detailed in ESM. 


\section{RESULTS}

PAH patients $(n=981)$

The mPAP ranged from 25 to $113 \mathrm{mmHg}$. The median sPAP/mPAP ratio was 1.591

$(98.3 \% \Phi)($ Table 2). This was consistent with the $\Phi$ hypothesis assuming a $<1 \%$ error in the estimation of median sPAP and mPAP values (Table 4), corresponding to $<0.8 \mathrm{mmHg}$ error for sPAP and $<0.5$ $\mathrm{mmHg}$ for mPAP. The mPAP/dPAP ratio was $1.559(96.3 \% \Phi)$. This was consistent with the hypothesis assuming a $<2 \%$ error in the estimation of $\mathrm{mPAP}$ and $\mathrm{dPAP}$, corresponding to $<1 \mathrm{mmHg}$ error for mPAP and $<0.6 \mathrm{mmHg}$ for dPAP.

The sPAP/mPAP ratio was similar in men (1.604) and women (1.583) $(P=0.08)$. The mPAP/dPAP was $2 \%$ lower in men (1.536) than in women (1.571) $(\mathrm{P}=0.01)$. Both the sPAP/mPAP and $\mathrm{mPAP} / \mathrm{dPAP}$ ratios were weakly related to age (Spearman's rho $=+0.27$ and +0.32 , respectively, each $P<0.01)$. There was a weak, negative relationship between the sPAP/mPAP and mPAP/dPAP ratios with mPAP ( $r$ o $=-0.23$ and -0.21 , resepectively, each $P<0.01$ ). The ratios slightly deviated from $\Phi$ at the highest mPAP quartile (Table 5), with the corresponding error in the estimation of the PA pressures entering the calculation of the two ratios being less than $4 \%$.

Assuming a $\pm 10 \%$ error in the measurement of PAPs, the individual values of sPAP/mPAP and mPAP/dPAP were consistent with the $\Phi$ hypothesis in 926 (94\%) and $813(83 \%)$ patients, respectively (Figure 2). Both ratios were consistent with the hypothesis in 794 (81\%) patients.

Historical controls with normal PAP $(n=44)$

The mPAP ranged from 9 to $20 \mathrm{mmHg}$. The mean sPAP/mPAP ratio was 1.572

$(97.2 \% \Phi)$ (Table 3). This was consistent with the $\Phi$ hypothesis assuming a $<2 \%$ error in the estimation of mean SPAP and mPAP values on average, with a corresponding $<0.4 \mathrm{mmHg}$ error for sPAP and $<0.3 \mathrm{mmHg}$ for mPAP. The mPAP/dPAP ratio was $1.470(90.9 \% \Phi)$, also consistent with the hypothesis assuming a $<5 \%$ error in the estimation of mPAP and dPAP, with a corresponding $<0.7$ 
$\mathrm{mmHg}$ error for mPAP and $<0.5 \mathrm{mmHg}$ for dPAP. Assuming a $<2 \mathrm{mmHg}$ error in the measurement of PAPs, the individual values of sPAP/mPAP and mPAP/dPAP were each consistent with the $\Phi$ hypothesis in $42 / 44$ subjects (95\%), (Figure 3). The ratios were both consistent with the hypothesis in $41 / 44$ subjects $(93 \%)$. 


\section{DISCUSSION}

The main result of our study was that sPAP/mPAP and mPAP/dPAP matched the golden ratio in a large population of incident, untreated PAH patients and in a smaller population of normotensive controls. This result is more than a mathematical peculiarity because the golden ratio allows linkage of three empirical observations on pulmonary hemodynamics that were hitherto apparently unrelated: namely the 0.61 slope of the mPAP vs sPAP relationship, the fact that PApp and mPAP have an almost 1:1 ratio, and the proportional relationship between SPAP, mPAP and dPAP.

To our knowledge this is the first study to test if the golden ratio applies in the pulmonary circulation. The golden ratio $\Phi$ is a proportion that has been found in many phenomena in nature $(7,8)$ and the potential contribution of $\Phi$ to numerous aspects of cardiovascular pathophysiology has been discussed (9-15). Because some authors have suggested that relevance of the golden ratio in biology may have been overstated in the past $(8,16,17)$, we first proposed a strong theoretical background to support the hypothesis that $\Phi$ may be a preferred number in the pulmonary circulation. Subsequently, we undertook an analysis of $981 \mathrm{PAH}$ patients (20), and 44 historical controls without PH to verify the relationship of $\Phi$ and the components of pulmonary artery pressure (18). Potential measurement errors were accounted for, as recommened by Markowsky (17). Indeed, human cardiovascular physiology is not mathematics and it is important to take into account both observational errors related to pressure measurements and the natural variability of biological variables $(16,17,25-27)$.

The sPAP/mPAP and mPAP/dPAP ratios matched $\Phi$ on average if one assumed a $<1 \mathrm{mmHg}$ error in the estimation of SPAP, MPAP and dAP on average. This error was deemed acceptable from a clinical point of view. As far as individual values are concerned, both sPAP/mPAP and mPAP/dPAP ratios demonstrated some dispersion around the golden ratio $\Phi$. This was fully expected in PAH because there is an up to $10 \%$ error related with the use of fluid-filled catheters (22). Similarly, the spontaneous short-term pressure variability of $8 \%(23)$ has been observed in controls without PH. 
Although the two ratios slightly decreased with mPAP, the individual values of the two ratios were consistent with the $\Phi$ hypothesis in the vast majority of PAH and controls. Nevertheless, we wish to emphasize the fact that it may be naïve or unrealistic to state that the $\Phi$ hypothesis applies in every one of these subjects. Rather, we conclude that the SPAP/mPAP and mPAP/dPAP ratios were constrained around $\Phi$ and that negative/positive individual errors cancel out on average.

Our group initially proposed that there is a linear relationship between mPAP and SPAP, with small pressure interecept of $2 \mathrm{mmHg}$ and 0.61 slope $(2,18)$, and this has been further confirmed by other groups $(5,24,28-31)$. A linear relationship between dPAP and mPAP, with near-zero intercept, was initially documented by Syyed et al. (24), with a slope of 0.71 . A recent study (30) has documented a slightly smaller proportionality constant $(\mathrm{dPAP} / \mathrm{mPAP}=0.62)$, a value supported by the present study $(1 / \Phi=0.618)$. Measurement of dPAP, especially when using fluid-filled catheters, may be subject to errors from catheter motion artifacts. This may explain the differences in the mPAP/dPAP ratio between studies, and the fact that mPAP/dPAP was more scattered than sPAP/mPAP in PAH (Figure 2). It is also possible that the mPAP/dPAP ratio may be less strongly constrained around $\Phi$ than SPAP/mPAP depending on the population under study, and this was especially noticeable in PAH with markedly elevated mPAP, and also in post-capillary PH patients (unpublished observation).

The pulmonary circulation is much more pulsatile than the systemic circulation. The oscillatory power amounts to about $25-30 \%$ of total power in the right ventricle vs $10-15 \%$ in the left ventricle (6). Pulse pressure is a higher proportion of mean pressure in the pulmonary artery than at the aortic level (6). The aortic systolic/mean pressure ratio and the mean aortic pressure/diastolic pressure ratio are in the 1.2 to 1.5 range (19), namely lower than $\Phi$, while sPAP/mPAP and mPAP/dPAP ratios matched $\Phi$. The diastolic pressure relative to systolic pressure is higher in the aorta (approximately $50-60 \%)(6,19)$ than in the pulmonary artery (approximately $38 \%=1 / \Phi^{2}$ ). The higher relative diastolic pressure in the aorta reflects the need of maintaining a high coronary 
perfusion pressure in diastole. Differences in the characteristics of right vs left ventricular ejection and differences in the resistive and elastic properties of the pulmonary vs systemic arterial trees may also explain the observed differences in arterial pulsatility between the two vascular beds (6).

The mPAP is determined by pulmonary vascular resistance (PVR), cardiac output and PA wedge pressure $(1,6)$. Pulse pressure strongly depends on stroke volume (SV) and on the PA compliance, which is most often estimated currently by the SV/PApp ratio $(32,33)$--Some authors have suggested that the PVR XSV/PApp product is constant in health and diseases and that such constancy may explain the fixed slope of the mPAP vs SPAP relationship $(33,34)$. One might argue that this explanation also applies to our results. However, we have recently challenged this hypothesis by documenting that the PVRXSV/PApp product is not constant in idiopathic PAH but is, on the contrary, as variable as patient mPAP (35).

The strength of our study was the large number of incident, untreated PAH patients included, and also the inclusion of normotensive controls. The main limitation was that patients with other forms of PH were not studied. Interestingly, if one tests our hypothesis in the largest hemodynamic database published on patients with chronic thromboembolic PH (CTEPH) undergoing pulmonary endarterectomy (36), both the mean sPAP/mPAP ratio and the mean mPAP/dPAP ratio are very close to $\Phi$ (Table 6). A large-scale study on CTEPH and postcapillary PH patients is in progress in our laboratory.

The implication of our study is that it may offer a new framework to describe the components of PA pressure based on the golden ratio, one of the "most astonishing numbers" (8). Unlike systemic hypertension, $\mathrm{PH}$ offers the unique opportunity to study hemodynamics over a more than tenfold prevailing pressure (mPAP 9-113 $\mathrm{mmHg}$ ). Over this large pressure range, the fluctuations in SPAP and dPAP around mPAP exhibited constant scaling factor matched to $\Phi$. In selfsimilar natural processes where $\Phi$ has been involved, it is believed to represent a way to maintain a state of minimal energy, as observed during growth (8). It must be noted, however, that a self-similar 
(fractal-like) process can hardly be involved in our results given the fixed time-scale. Thus, the biological reasons behind the presence of $\Phi$ in the pulmonary circulation remain to be determined. Further studies are needed to clarify whether the golden ratio reflects "only a fascinating prevalent tendency" (37) of the pulmonary circulation or may have clinical implications. It remains to be established whether patients with $\mathrm{SPAP} / \mathrm{mPAP}$ and $\mathrm{mPAP} / \mathrm{dPAP}$ ratios markedly deviating from this "golden behavior" may exhibit some specific features in terms of cause of PH or future cardiovascular events including right heart failure.

In conclusion, in incident untreated PAH patients and in controls, the fluctuations in sPAP and dPAP around mPAP exhibited constant scaling factor shown to be the golden ratio. This may provide a new theoretical background to link together various empirical observations on pulmonary hemodynamics that were hitherto apparently unrelated.This remarkable property of pulmonary circulation warrants further confirmation in other types of $\mathrm{PH}$ and warrants explanation. 


\section{Authors contribution}

Denis Chemla and David Boulate conceived the study.

Jason Weatherald, Edmund MT Lau, Laurent Savale, David Montani and Philippe Hervé performed the right heart catheterization.

Denis Chemla, David Boulate, Jason Weatherald and Edmund Lau performed the calculations and statistical analysis.

Denis Chemla, David Boulate, Jason Weatherald, Edmund Lau and Philippe Hervé wrote the manuscript.

All authors discussed the results and have read and improved the final manuscript.

\section{Acknowledgements}

The authors thank Kaixian Zhu for helpful discussion during the preparation of the manuscript. 


\section{REFERENCES}

1- Galiè N, Humbert M, Vachiery J-L, Gibbs S, Lang I, Torbicki A, Simonneau G, Peacock A, Vonk Noordegraaf A, Beghetti M, Ghofrani A, Gomez Sanchez MA, Hansmann G, Klepetko W, Lancellotti P, Matucci M, McDonagh T, Pierard LA, Trindade PT, Zompatori M, Hoeper M, Aboyans V, Vaz Carneiro A, Achenbach S, Agewall S, Allanore Y, Asteggiano R, Paolo Badano L, Albert Barberà J, Bouvaist H, Bueno H, Byrne RA, Carerj S, Castro G, Erol Ç, Falk V, FunckBrentano C, Gorenflo M, Granton J, lung B, Kiely DG, Kirchhof P, Kjellstrom B, Landmesser U, Lekakis J, Lionis C, Lip GYH, Orfanos SE, Park MH, Piepoli MF, Ponikowski P, Revel M-P, Rigau D, Rosenkranz S, Völler H, Luis Zamorano J. 2015 ESC/ERS Guidelines for the diagnosis and treatment of pulmonary hypertension: The Joint Task Force for the Diagnosis and Treatment of Pulmonary Hypertension of the European Society of Cardiology (ESC) and the European Respiratory Society (ERS): Endorsed by: Association for European Paediatric and Congenital Cardiology (AEPC), International Society for Heart and Lung Transplantation (ISHLT). Eur Heart J. 2016;37: 67-119. doi: 10.1093/eurheartj/ehv317. Epub 2015 Aug 29.

2- Chemla D, Castelain V, Humbert M, Hébert JL, Simonneau G, Hervé P. New formula for predicting mean pulmonary artery pressure using systolic pulmonary artery pressure. Chest. 2004; 126: 1313-1317.

3- Chemla D, Humbert M, Sitbon O, Montani D, Hervé P. Systolic and mean pulmonary artery pressures: are they interchangeable in patients with pulmonary hypertension? Chest. 2015; 147: $943-950$.

4- Aduen JF, Castello R, Daniels JT, Diaz JA, Safford RE, Heckman MG, Crook JE, Burger CD. Accuracy and precision of three echocardiographic methods for estimating mean pulmonary artery pressure. Chest. 2011; 139: 347-352.

5- Amsallem M, Sternbach JM, Adigopula S, Kobayashi Y, Vu TA, Zamanian R, Liang D, Dhillon G, Schnittger I, McConnell MV, Haddad F. Addressing the controversy of estimating pulmonary arterial pressure by echocardiography. J Am Soc Echocardiogr. 2016; 29: 93-102.

6- Milnor WR. Cardiovascular physiology. 1990. Oxford University Press, New York. 215 pp.

7- Ghyka MC. The Golden Number: Pythagorean Rites and Rhythms in the Development of Western Civilization. 2016. Inner Traditions Bear and Company, USA.

8- Livio M. The golden ratio: The story of Phi, the world's most astonishing number. 2003. Broadway Books, New York, NY, USA

9- Gibson CM, Gibson WJ, Murphy SA, Marble SJ, McCabe CH, Turakhia MP, Kirtane AJ, Karha J, Aroesty JM, Giugliano RP, Antman EM; TIMI Study Group. Association of the Fibonacci 
cascade with the distribution of coronary artery lesions responsible for ST-segment elevation myocardial infarction. Am J Cardiol. 2003; 92: 595-7.

10- Ulmer H, Kelleher CC, Dünser MW. George Clooney, the cauliflower, the cardiologist, and phi, the golden ratio. BMJ 2009; 339: b4745

11- Henein MY; Golden Ratio Collaborators, Zhao Y, Nicoll R, Sun L, Khir AW, Franklin K, Lindqvist P. The human heart: application of the golden ratio and angle. Int J Cardiol. 2011; 150: 23942.

12- Ashrafian H, Athanasiou T. Fibonacci series and coronary anatomy. Heart Lung Circ. 2011; 20: 483-4.

13- Yetkin G, Sivri N, Yalta K, Yetkin E. Golden Ratio is beating in our heart. Int J Cardiol. 2013; 168: 4926-7.

14- Yetkin E, Topbaş U, Yanik A, Yetkin G. Does systolic and diastolic blood pressure follow Golden Ratio? Int J Cardiol. 2014; 176: 1457-9.

15- Papaioannou TG, Gialafos E, Karamanou M, Tsoucalas G, Tousoulis D. The 'Divine' or 'Golden' Arterial Pulse. Eur Heart J. 2017; 38: 2925-2928.

16- Iosa M, Morone G, Paolucci S. Phi in physiology, psychology and biomechanics: The golden ratio between myth and science. Biosystems. 2018; 165:31-39.

17- Markowsky G. Misconceptions about the golden ratio. The College Mathematic Journal 1992; 23: $2-19$

18- Chemla D, Castelain V, Provencher S, Humbert M, Simonneau G, Hervé P. Evaluation of various empirical formulas for estimating mean pulmonary artery pressure by using systolic pulmonary artery pressure in adults. Chest. 2009; 135: 760-768.

19- Chemla D, Antony I, Zamani K, Nitenberg A. Mean aortic pressure is the geometric mean of systolic and diastolic aortic pressure in resting humans. J Appl Physiol. 2005; 99: 2278-84.

20- Weatherald J, Boucly A, Chemla D, Savale L, Peng M, Jevnikar M, Jaïs X, Taniguchi Y, O'Connell C, Parent F, Sattler C, Hervé P, Simonneau G, Montani D, Humbert M, Adir Y, Sitbon O. The prognostic value of follow-up hemodynamic variables after initial management in pulmonary arterial hypertension. Circulation 2018; 137: 693-704.

21- Curtiss EI, Reddy PS, O'Toole JD, Shaver JA. Alterations of right ventricular systolic time intervals by chronic pressure and volume overloading. Circulation. 1976; 53: 997-1003.

22- Laskey WK, Ferrari VA, Palevsky HI, Kussmaul WG.Pulmonary artery hemodynamics in primary pulmonary hypertension. J Am Coll Cardiol. 1993; 21: 406-12.

23- Reynolds DW1, Bartelt N, Taepke R, Bennett TD. Measurement of pulmonary artery diastolic pressure from the right ventricle. J Am Coll Cardiol. 1995; 25: 1176-82. 
24- Syyed R, Reeves JT, Welsh D, Raeside D, Johson MK, Peacock AJ. The relationship between the components of pulmonary artery pressure remains constant under all conditions in both health and disease. Chest 2008; 133: 633-639

25- Mirams GR, Pathmanathan P, Gray RA, Challenor P, Clayton RH. Uncertainty and variability in computational and mathematical models of cardiac physiology. J Physiol. 2016; 594:68336847.

26- Falsetti HL, Mates RE, Carroll RJ, Gupta RL, Bell AC. Analysis and correction of pressure distorsion in fluid-filled catheter systems. Circulation 1974; 49: 165-172

27- Rich SR, d'Alonzo GE, Dantzker DR, Levy PS. Magnitude and implications of spontaneous hemodynamic variability in primary pulmonary hypertension. Am J Cardiol 1985; 55: 159-63

28- Steckelberg RC, Tseng AS, Nishimura R, Ommen S, Sorajja P. Derivation of mean pulmonary artery pressure from noninvasive parameters. J Am Soc Echocardiogr 2013; 26: 464-8.

29- Miller WL, Grill DE, Borlaug BA. Clinical features, hemodynamics, and outcomes of pulmonary hypertension due to chronic heart failure with reduced ejection fraction. JACC: Heart Failure 2013; $1: 290-9$

30- Handoko ML, De Man FS, Oosterveer FP, Bogaard HJ, Vonk-Noordegraaf A, Westerhof N. A critical appraisal of transpulmonary and diastolic pressure gradients. Physiol Rep. 2016; 4: e12910.

31- Vanden Eynden F, Racapé J, Vincent J, Vachiéry JL, Bové T, Van Nooten G.The linear relationship between systolic pulmonary artery pressure and mean pulmonary artery pressure is maintained regardless of autonomic or rhythm disturbances. Respir Res. 2016; 17: 33.

32- Chemla D, Lau E, Papelier Y, Attal P, Hervé P. Pulmonary vascular resistance and compliance relationship in pulmonary hypertension. Eur Respir J 2015; 46: 1178-1189

33- Saouti N, Westerhof N, Postmus PE, Vonk-Noordegraaf A. The arterial load in pulmonary hypertension. Eur Respir J 2010; 19: 197-203

34- Kind T, Faes TJ, Vonk-Noordegraaf A, Westerhof N. Proportional relationship between systolic, diastolic and mean pulmonary artery pressure are explained by vascular properties. Cardiovasc Eng Technol 2011; 2: 15-23

35- Chemla D, Weatherald JC, Lau E, Savale L, Boucly A, Attal P, Jaïs X, Parent F, Girerd B, Simonneau G, Montani D, Humbert M, Sitbon O, Hervé P. Clinical and hemodynamic correlates of pulmonary arterial stiffness in incident, untreated patients with idiopathic pulmonary arterial hypertension. Chest 2018; 154 (4): 882-892. 
36- Madani MM, Auger WR, Pretorius V, Sakakibara N, Kerr KM, Kim NH, Fedullo PF, Jamieson SW.Pulmonary endarterectomy: recent changes in a single institution's experience of more than 2,700 patients. Ann Thorac Surg. 2012; 94(1):97-103.

37- Coxeter HSM. The golden section, phyllotaxis, and Wythoff's game. Scripta Mathematica 1953; 19: 135-143 


\section{Legends of the Figures}

Figure 1. The golden ratio hypothesis. Pulmonary artery pressure is represented as as a function of time. Systolic (sPAP), mean (mPAP) and diastolic (dPAP) pulmonary artery pressures are indicated. The hypothesis that the SPAP/mPAP ratio and the mPAP/dPAP ratio matched the golden ratio (Phi, $\Phi=1.618$ ) was tested. The proportionality between pulmonary artery pressure components means that as sPAP is to mPAP (sPAP/mPAP), so is mPAP to dPAP (mPAP/dPAP), with the proportionality constant matched to $\Phi$ according to our study hypothesis.

Figure 2. Fluid-filled pulmonary pressures in incident untreated patients with pulmonary arterial hypertension PAH ( $n=981)$. Upper panel: scatter diagram presenting the relationship between mean PA pressure (mPAP) and the systolic PA pressure (sPAP) over mPAP ratio. When the possibility of a $\pm 10 \%$ error in individual pressure measurements was accounted for, sPAP/mPAP values ranging from $0.82 \Phi$ to $1.22 \Phi$ (dotted lines) were consistent with the hypothesis. Accordingly, 926 patients (94\%) had their SPAP/mPAP ratio within this range. Lower panel: scatter diagram presenting the relationship between mPAP and the mPAP over diastolic PA pressure (dPAP) ratio. 813 patients ( $83 \%)$ had their mPAP/dPAP ratio within the 0.82 to $1.22 \Phi$ range. Overall, the sPAP/mPAP and $\mathrm{mPAP} / \mathrm{dPAP}$ ratios were both consistent with the $\Phi$ hypothesis in 794 (81\%) patients.

Figure 3. High-fidelity pulmonary pressures in historical controls $(n=44)$. Upper panel: scatter diagram presenting the relationship between mean PA pressure (mPAP) and the systolic PA pressure (sPAP) over mPAP ratio. Values of the sPAP/mPAP ratio ranging from $0.80 \Phi$ to $1.27 \Phi$ (dotted lines) were consistent with the $\Phi$ hypothesis, if one assumed $a<2 \mathrm{mmHg}$ error in individual pressure peasurements ( $n=42 / 44$ subjects, $95 \%)$. Lower panel: scatter diagram presenting the relationship between mPAP and the mPAP over diastolic PA pressure (dPAP) ratio. If one assumes a $<2 \mathrm{mmHg}$ error, values of the mPAP/dPAP ratio ranging 0.71-1.43 $\Phi$ (dotted lines) were consistent with the hypothesis in 42/44 subjects (95\%). The SPAP/mPAP and mPAP/dPAP ratios were both consistent with the $\Phi$ hypothesis in $41 / 44$ subjects (93\%). 
Golden Ratio Hypothesis

$$
\mathrm{a} / \mathrm{b}=\mathrm{b} / \mathrm{c}=\Phi=1.618
$$

\section{Pulmonary artery pressure}
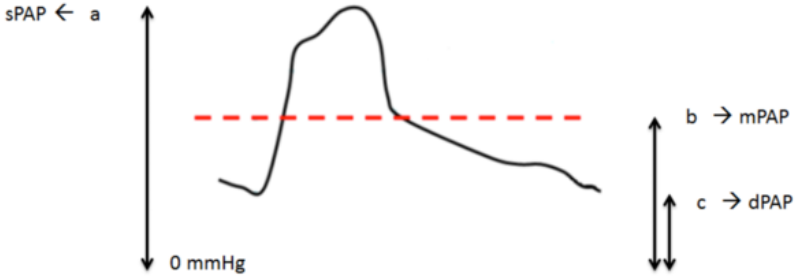


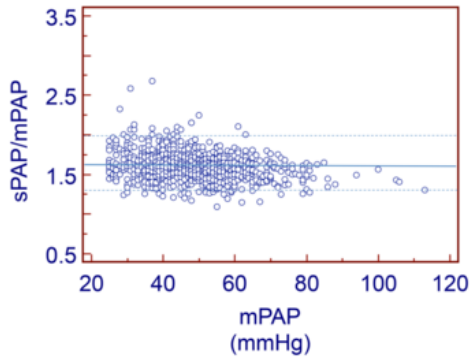

$\Phi$

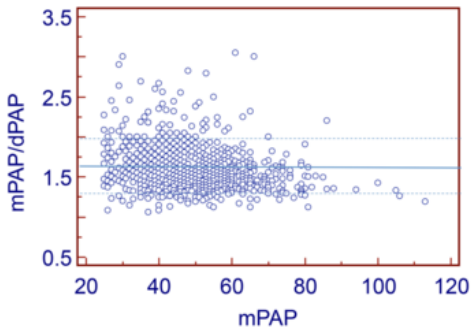

$\Phi$

$(\mathrm{mmHg})$ 

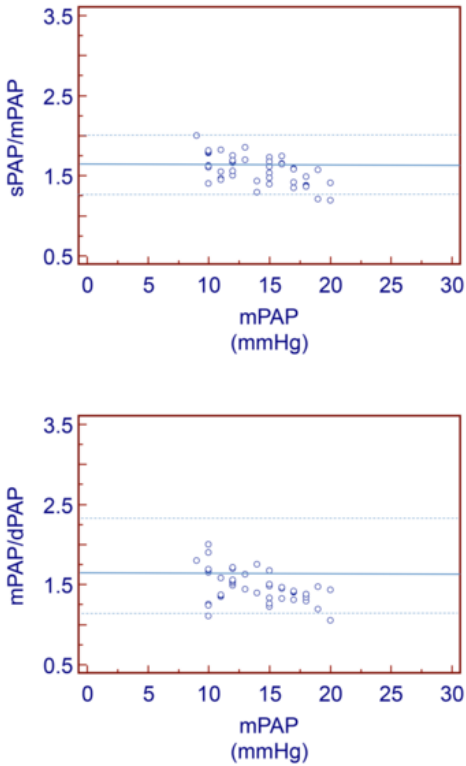

$\Phi$ 
Table 1- Baseline characteristics in PAH patients ( $n=981)$

\begin{tabular}{lll}
\hline Variable & 581 & $(59.2 \%)$ \\
\hline Female Gender & 63 & $(48-74)$ \\
Age (y) & 1.79 & $(1.65-1.95)$ \\
Body Surface Area $\left(\mathrm{m}^{2}\right)$ & & \\
Etiology of PAH & 748 & $(76.2 \%)$ \\
Idiopathic & 152 & $(15.5 \%)$ \\
Drugs and Toxins & 81 & $(8.3 \%)$ \\
Heritable & 101 & $(12.5 \%)$ \\
Positive vasoreactivity test (n=807) & & \\
NYHA FC (n=967) & & $(15.5 \%)$ \\
I & 319 & $(2.6 \%)$ \\
II & 25 & $(22.1 \%)$ \\
III & 578 & $(59.8 \%)$ \\
IV & & \\
\end{tabular}

Variables are expressed as number (\%), median (Interquartile range $25-75 \%$ ) or mean \pm standard deviation. PAH - pulmonary arterial hypertension; NYHA FC - New York Heart Association functional class; 6MWD - 6-minute walk distance. 
Table 2. Hemodynamic variables in PAH patients $(n=981)$

\begin{tabular}{|l|c|}
\hline & \\
\hline sPAP, mmHg & $76(64-89)$ \\
\hline mPAP, mmHg & $47(40-56)$ \\
\hline dPAP, mmHg & $30(24-37)$ \\
\hline sPAP/mPAP (-) & $1.591(1.500-1.693)$ \\
\hline mPAP/dPAP (-) & $1.559(1.432-1.720)$ \\
\hline PApp / mPAP (-) & $0.95(0.82-1.10)$ \\
\hline Cl, L/min/m ${ }^{2}$ & $2.35(1.91-2.84)$ \\
\hline HR, bpm & $80(69-90)$ \\
\hline SVI, mL/m ${ }^{2}$ & $31(24-38)$ \\
\hline PAWP, mmHg & $9(7-12)$ \\
\hline PVR, WU & $9.0(6.2-12.7)$ \\
\hline RAP, mmHg (n=943) & $8(5-12)$ \\
\hline
\end{tabular}

Values are median (IQR) except for gender (number (\%)). Cl: cardiac index. dPAP: diastolic pulmonary artery pressure. HR: heart rate. MPAP: mean pulmonary artery pressure. PAP: pulmonary artery pressure. PApp $=$ Pulmonary artery pulse pressure (PApp $=$ sPAP - dPAP). PAWP: Pulmonary artery wedge pressure. PVR: pulmonary vascular resistance. SPAP: systolic pulmonary artery pressure. SVI: stroke volume index. WU: Wood units. All pressure measurements obtained from fluid-filled catheters. The geometric mean of SPAP and dPAP gave an accurate and precise estimate of mPAP (mean bias $0.2 \mathrm{mmHg}$, SD $3.1 \mathrm{mmHg}$ ). Note that the sum of dPAP+mPAP (77 $\mathrm{mmHg}$ ) was only $1 \mathrm{mmHg}$ higher than SPAP. 
Table 3. Clinical and hemodynamic variables in 44 normotensive controls (mPAP $\leq 20 \mathrm{mmHg}$ ).

\begin{tabular}{|l|c|}
\hline & \\
\hline Age, years & $43(16)$ \\
\hline Female gender & $16(36)$ \\
\hline sPAP, mmHg & $22(4)$ \\
\hline mPAP, mmHg & $14(3)$ \\
\hline dPAP, mmHg & $10(3)$ \\
\hline SPAP/mPAP (-) & $1.572(0.178)$ \\
\hline mPAP/dPAP (-) & $1.470(0.204)$ \\
\hline PApp / mPAP (-) & $0.88(0.23)$ \\
\hline
\end{tabular}

Values are means (SD) except for gender (number (\%)). Data extracted from our systematic review (18) involving 5 high-fidelity pressure studies (Refs 2 and 21-24). dPAP: diastolic pulmonary artery pressure. mPAP: mean pulmonary artery pressure. PAP: pulmonary artery pressure. $\mathrm{PApp}=$ Pulmonary artery pulse pressure (PApp $=\mathrm{sPAP}-\mathrm{dPAP})$. The geometric mean of sPAP and dPAP gave an accurate and precise estimate of mPAP (mean bias $0.5 \mathrm{mmHg}, \mathrm{SD} 0.9 \mathrm{mmHg}$ ). Note that the sum of dPAP+mPAP (24 mmHg) was only $2 \mathrm{mmHg}$ higher than sPAP. 
Table 4. Sensitivity analysis

\begin{tabular}{|l|c|c|c|}
\hline $\begin{array}{l}\text { \% error in the } \\
\text { estimation of PAP }\end{array}$ & $\begin{array}{c}\text { Range of sPAP/mPAP and } \\
\text { mPAP/dPAP values consistent } \\
\text { with the } \Phi \text { hypothesis }\end{array}$ & Correction factor & Correction factor \\
(lower limit) & (upper limit) \\
\hline $\mathbf{1 \%}$ & $1.585-1.650$ & $0.98 \times \Phi$ & $1.02 \times \Phi$ \\
\hline $\mathbf{2 \%}$ & $1.554-1.684$ & $(0.99 / 1.01)$ & $(1.01 / 0.99)$ \\
\hline $\mathbf{5 \%}$ & $1.464-1.788$ & $0.96 \times \Phi$ & $1.04 \times \Phi$ \\
& $1.324-1.977$ & $0.90 \times \Phi$ & $(1.02 / 0.98)$ \\
\hline $\mathbf{1 0 \%}$ & $(0.95 / 1.05)$ & $1.10 \times \Phi$ \\
& & $0.82 \times \Phi$ & $1.05 / 0.95)$ \\
\hline
\end{tabular}

The sensitivity analysis was performed using the Markowsky's method (17). $\Phi=$ golden ratio = 1.618 . The unavoidable sources of error in the estimation of PA pressures due to measurement error were taken into account. This analysis was applied to median/mean values of the SPAP/mPAP and $\mathrm{mPAP} / \mathrm{dPAP}$ ratios in the whole study population, and to individual values of the ratios in PAH patients. Concerning individual values of the ratios in control subjects whose PAP values were normally low, the Markoswy's method was applied using a fixed $<2 \mathrm{mmHg}$ error, as explained in ESM. 


\section{Table 5}

Testing the $\Phi$ hypothesis throughout the mean pulmonary artery pressure range in incident, untreated pulmonary arterial hypertension patients $(\mathrm{n}=981)$.

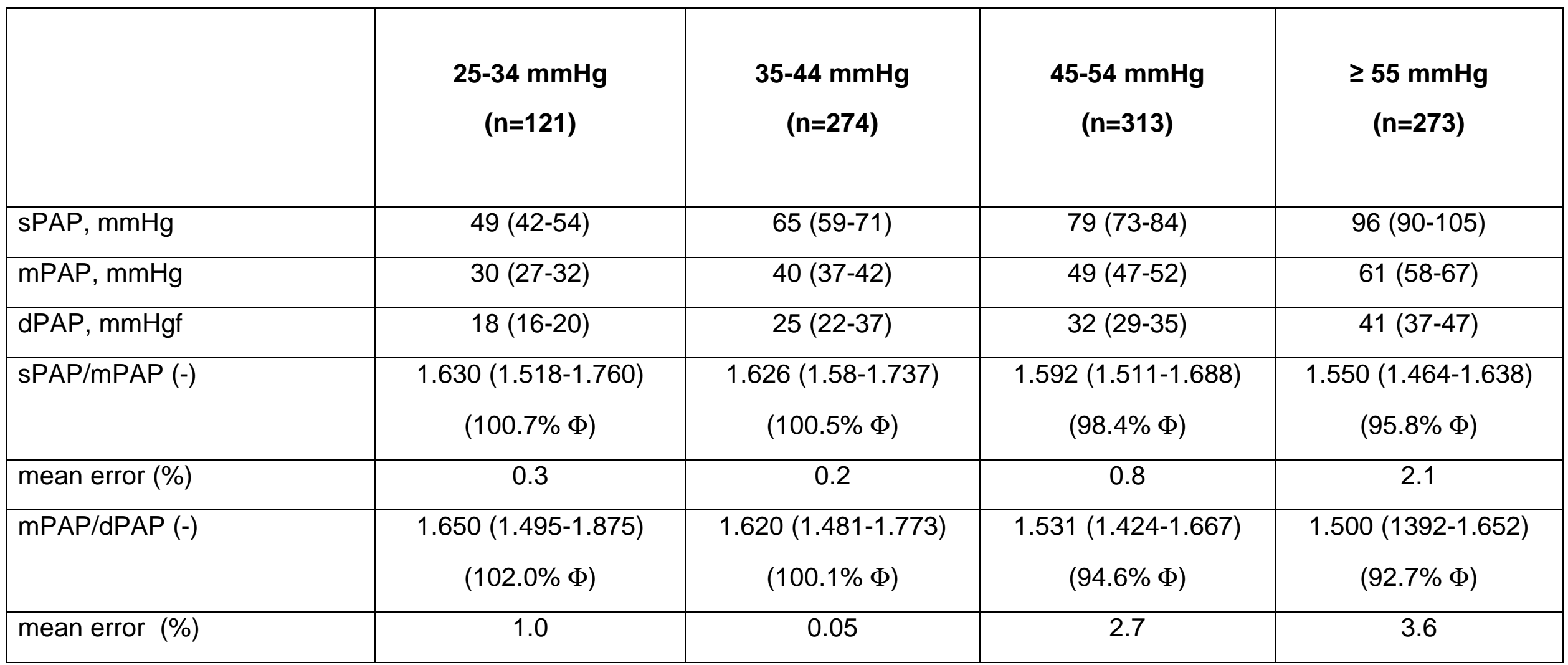

Median (IQR) or \% are indicated. Same abbreviations as in Table 1. $\Phi$ : the golden ratio (1.618). To be consistent with the hypothesis that the ratio under study matches $\Phi$, the corresponding mean error in the estimation of the PA pressures entering the calculation of the two ratios is indicated, according to the Markowsky's method (17). 


\section{Table 6}

Testing the $\Phi$ hypothesis in the largest database published on chronic thromboembolic pulmonary hypertension (CTEPH) patients by Madani et al (Ref 36).

\begin{tabular}{|c|c|c|c|c|}
\hline & Preoperative & Preoperative & Postoperative & Postoperative \\
\hline & $\begin{array}{l}\text { Group 1 } \\
(n=1,000)\end{array}$ & $\begin{array}{l}\text { Group } 2 \\
(n=500)\end{array}$ & $\begin{array}{l}\text { Group 1 } \\
(n=1,000)\end{array}$ & $\begin{array}{l}\text { Group } 2 \\
(n=500)\end{array}$ \\
\hline $\mathrm{mPAP}, \mathrm{mmHg}$ & $46.1(11.4)$ & $45.5(11.6)$ & $28.7(10.1)$ & $26.0(8.4)$ \\
\hline calculated mean mPAP/dPAP & $\begin{array}{c}1.623 \\
(100.3 \% \Phi)\end{array}$ & $\begin{array}{c}1.667 \\
(103.0 \% \Phi)\end{array}$ & $\mathrm{N} / \mathrm{A}$ & $\mathrm{N} / \mathrm{A}$ \\
\hline
\end{tabular}

Group 1: CTEPH patients included between March 1999 and October 2006.

Group 2: CTEPH patients included between October 2006 and December 2010.

Pressure data are given as indicated in Table 1 and Table 3 of Ref 36, where mean (SD) is indicated.

From these published values we calculated the mean SPAP/mPAP ratio and the mPAP/dPAP ratio. N/A: not available. 